\title{
Spontaneous Reporting on Adverse Events by Consumers in the United States: An Analysis of the Food and Drug Administration Adverse Event Reporting System Database
}

\author{
Tadashi Toki ${ }^{1} \cdot$ Shunsuke Ono ${ }^{1}$ (D)
}

Published online: 3 May 2018

(c) The Author(s) 2018

\begin{abstract}
Background Voluntary reports on adverse events (AEs) submitted by consumers have been facilitated through the MedWatch program in the United States (US), but few studies have described the characteristics of voluntary reports.

Objective The aim of this study was to reveal the characteristics of current voluntary reports on AEs reported by consumers and healthcare professionals.

Methods We performed analysis on voluntary reports of AEs in the US Food and Drug Administration AE Reporting System (FAERS) database submitted in 2016. We compared reports by consumers with those by healthcare professionals.

Results The number of voluntary reports by consumers has increased since 2013 in the US. Reports by consumers were different from ones by health professionals in several important aspects such as demographics and outcomes of patients, AEs, and suspect drugs. The proportion of reports on female patients and on disability as a patient outcome were higher in reports by consumers than in those by healthcare professionals. Consumers more frequently reported concomitant drugs compared with healthcare professionals. Time to report varied among the occupations and depending on seriousness of outcomes.
\end{abstract}

Electronic supplementary material The online version of this article (https://doi.org/10.1007/s40801-018-0134-0) contains supplementary material, which is available to authorized users.

Shunsuke Ono

shun-ono@mol.f.u-tokyo.ac.jp

1 Laboratory of Pharmaceutical Regulation and Sciences, Graduate School of Pharmaceutical Sciences, The University of Tokyo, 7-3-1 Hongo, Bunkyo-ku, Tokyo 113-0033, Japan
Conclusions Our analysis of voluntary AE reports in the US FAERS database has shown that voluntary reports tended to include AEs related to subjective symptoms, as in some previous studies on patient reporting in the EU. Voluntary reports by consumers seemed to be different from ones by healthcare professionals in important aspects including demographics and reporting behaviors. These findings suggest that the heterogeneities should be addressed appropriately in using spontaneous reports.

\section{Key Points}

The number of voluntary adverse event (AE) reports by consumers, which reflect concerns and restrictions specific to consumers, has apparently increased since the introduction of the 'consumerfriendly' reporting form FDA3500B in 2013, accounting for about half of the total AE reports in the second quarter of 2016.

Reports by consumers were different from ones by health professionals in important aspects such as demographics and outcomes of patients, AEs, and suspect drugs. Report completeness and time-toreport also varied depending on the occupation of reporters.

Observed characteristics in spontaneous reporting in the US should be considered in using AE reports in pharmacovigilance activities, especially when $\mathrm{AE}$ reports are compared with ones in different countries/regions. 


\section{Introduction}

Recent changes in pharmaceutical markets and regulations, including introduction of new accelerated approval pathways [1-3], new lines of therapies with innovative pharmacological mechanisms from molecular-targeted drugs to immune checkpoint inhibitors and to cell therapies, and growing expectations from patients for new therapies under development, have prompted regulators and industries to introduce new drugs faster and more efficiently [4]. These trends have made postmarketing pharmacovigilance more important than ever [5]. The United States (US) Food and Drug Administration (FDA) Adverse Event (AE) Reporting System (FAERS) database, one of the largest pharmacovigilance databases, plays a key role in both collecting and providing data on drug-related AEs. In the US, the FAERS database gives the FDA critical signals and a decision basis to take regulatory actions such as ordering labeling changes in the warning and/or precaution sections to improve drug use in markets [6,7]. Epidemiologists worldwide use the database to detect novel drug-related safety events, to identify possible mechanisms of AEs, and to explore efficient methods to detect potential drug-related AEs [8-14]. The database has been used beyond the realm of safety. For example, several researchers have recently studied drug-drug interactions with new approaches using the FAERS database, which could lead to discovery of promising new concomitant uses of drugs in certain therapeutic areas [15, 16].

For all purposes, the integrity of databases is a critical condition for users to obtain unbiased conclusions. Underreporting has been a serious issue that afflicts pharmacovigilance activities worldwide [6, 17]. Previous studies showed that under-reporting was caused by many factors, including inevitable dependency on reporters volunteering incentives and lack of awareness of how to use public reporting systems, or even their existence [18, 19]. A practical approach to improve the situation of under-reporting is to publicize the reporting system and to encourage not only healthcare professionals but also the consumers who actually experience AEs, and their families, to submit AE reports to the FDA. The FDA has also made efforts to reduce undesirable disproportionality (e.g., over- or under-representation of specific populations) in the FAERS. To alleviate these concerns, the FDA has continuously expanded the MedWatch program for more than 20 years. The FDA introduced the first voluntary reporting form FDA3500 in 1993 and the form FDA3500B in 2013, a voluntary consumer-friendly reporting form, to encourage reporting by patients $[20,21]$.

It is important for database users to acknowledge basic characteristics of spontaneous safety databases, including types and variations of data sources, because the internal and external validity of analyses using the databases totally depend on them. Regarding that perspective, however, spontaneous reports have attracted less attention than obligatory reports from the industry. There are a couple of studies on spontaneous reports by nurses and physicians in locally established databases of AE reports, but there have been few analytical studies focusing on spontaneous reporting by consumers in the US [22-25]. In the European Union (EU), patient-oriented reporting has grown gradually since the 2000s, and current situations have been described in previous studies [25-30]. In one study it was concluded that patient reporting successfully complemented reporting by healthcare professionals, and the conclusion was consistent with that of a study in the UK $[31,32]$. Another study using the database of the Dutch Pharmacovigilance Center Lareb showed that patients report clinical information at a similar level to healthcare professionals.

In this study, we aimed to identify the characteristics of recent voluntary reports submitted by consumers and compare them with those by healthcare professionals. We discussed what backgrounds have led to the observed differences, especially focusing on how information available on AEs could influence reporters' attitudes to AE reporting.

\section{Methods}

We analyzed the FAERS database and examined the reports submitted by consumers and healthcare professionals. We showed the transition of the numbers of voluntary reports in the US (Fig. 1), and conducted several descriptive analyses to reveal the characteristics of current voluntary reports. All the descriptive analyses were based on the reports $(25,814$ reports) in the first and second quarter of 2016, and the analyses related to primary suspect drugs were based on reports for which primary suspect drugs were registered in the SIDER4.1 database.

\subsection{Food and Drug Administration (FDA) Adverse Event (AE) Reporting System (FAERS) Data Preparation}

We used the JAPIC AERS database, comprising the FAERS database cleaned by the Japan Pharmaceutical Information Center (JAPIC), which was provided for our study under a collaborative research contract. During data cleaning, JAPIC eliminated redundant cases, adjusted units to make them uniform, mapped drug names onto their drug name dictionary, and refreshed preferred terms (PTs) in the Medical Dictionary for Regulatory Activities (MedDRA, ver.19.1) terminology. 


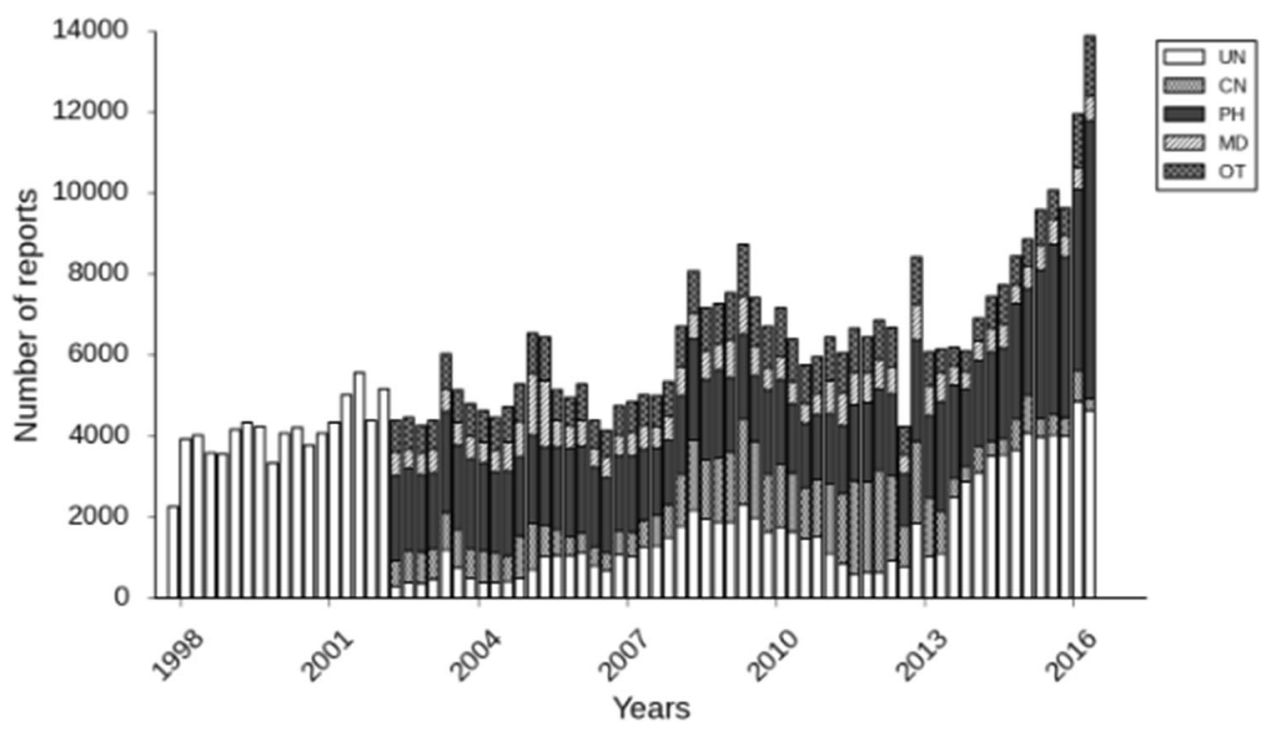

Fig. 1 Changes in the number of voluntary reports by consumers and healthcare professionals. The bar graph shows the number of quarterly reports. The 'only-for-consumer' FDA3500B form was introduced in 2013, and AE reports by unknown occupation reporters (UN) have increased sharply since the 3rd quarter (Q3) of 2013,

\subsection{Definition of Reporters}

We classified reporters into consumers, pharmacists, physicians, and other healthcare professionals according to the reporter's occupation on the form. The main focus of this research was to reveal the characteristics of voluntary reports (e.g., demographics, background diseases, type of AEs, time-to-report) by consumers in the US and compare them with reports by healthcare professionals. For this aim, we looked at both the voluntary reports by those who clearly identified themselves as 'consumers' in the classical voluntary reporting form FDA3500 (Group 1) and the voluntary reports in form FDA3500B that lacked the occupation item because it is intended for use by consumers (Group 2). The form FDA3500B, which was released in 2013 to facilitate consumers' voluntary reports, has the same items as FDA3500 except for occupation. The instructions in FDA3500B are easier for consumers to understand, even for first-time users.

Although the reports in both groups (1 and 2) were supposed to be submitted by consumers, another research question would be on whether the users of the form FDA3500 might be different from the users of the form FDA3500B in some demographic traits as well as AEs and drugs reported. However, the current dataset publicly available from FAERS does not have a code showing in which form the report was submitted and it was impossible to compare Groups 1 and 2 directly. We therefore tagged Group 1 as the reports by 'consumers' and all the reports that lacked reporter's occupation, including Group 2, as which suggests that most UNs after Q3 of 2013 are consumers using the FDA3500B form. $A E$ adverse event, $C N$ consumer, $M D$ physician, $O T$ other healthcare professional, $P H$ pharmacist, $U N$ unknown occupation reporter

reports by 'unknown occupation' reporters. We used the following abbreviations for each type of reporter: CNs for consumers, UNs for unknown occupation reporters (including consumers using FDA3500B), PHs for pharmacists, MDs for physicians, and OTs for other healthcare professionals.

\subsection{Additional Data Collection}

To determine whether AEs in a report were already known (i.e., written in the labels at the time of AE reporting), we used the SIDER database on marketed chemical medicines and related adverse drug reactions from drug labels [33]. SIDER used the MedDRA dictionary to extract side effects from labels. The results of this mapping are available under a Creative Commons Attribution-Noncommercial-Share Alike 4.0 License. We downloaded the data from the SIDER website on April 18, 2017. Because SIDER version 4.1 was released on October 21, 2015, we treated the adverse drug reactions (ADRs) included in SIDER 4.1 as known ADRs. If one of the AEs in a report was a known ADR for the primary suspect drug in the report, we considered that the report had known ADR(s). We collected data on safety labeling changes from monthly safety labeling changes on the FDA's MedWatch websites [34]. Using the archival data we also obtained data on how many times the primary suspect drug experienced safety labeling changes in the black box warning, warning, and/or precaution sections. 


\subsection{Data Analysis}

The completeness of reports is a quality indicator that reflects the characteristics of reporters and environments, including reporters' motivation for $\mathrm{AE}$ reporting. We assessed the completeness in reports by different types of reporters for the following items: patient sex, patient age, patient weight, indications, event date, route of primary suspect drug administration, secondary suspect drug(s), and concomitant drugs. We examined the time to report (i.e., the time between $\mathrm{AE}$ occurrence and submission of the $\mathrm{AE}$ report to the FDA) because it is an interesting indicator reflecting reporters' responsiveness to AE reporting. This indicator reflects various reporting conditions, including carefulness, to determine the cause of AEs and priority of $\mathrm{AE}$ reporting, which might be especially notable for healthcare professionals. We analyzed the time to report from two aspects. First, we examined possible associations between the time to report and whether the AEs were known at the time of AE occurrence in all cases, ones with serious outcome, or ones with non-serious outcome. This would offer clues to how responsiveness differs between the reporters facing different levels of uncertainties. Second, we examined time to report by stratifying by whether the primary suspect drug had experienced safety labeling changes, which may raise public awareness of drugs and AEs and affect healthcare professionals and consumers in different ways.

We used PostgreSQL version 9.3 and Python version 2.7 for data extraction. Chi square test and Wilcoxon rank-sum test were used for inter-group comparisons. The significance threshold was set at $p=0.05$ in all statistical analyses.

\section{Results}

\subsection{Trend in Spontaneous AE Reporting}

From Q4 of 1997 through Q2 of 2016, 482,938 voluntary reports were submitted to the FDA, 69,267 by consumers, 175,675 by unknown occupation reporters, 138,454 by pharmacists, 40,668 by physicians, and 58,874 by other healthcare professionals. The total number of voluntary reports increased from 2251 in Q4 of 1997 to 13,866 in Q2 of 2016, with the upward trend especially noticeable since Q3 of 2013 when the consumer-friendly form FDA3500B became an option for consumer reporting (Fig. 1). Because the number of reports from consumers using the form FDA3500 diminished after 2013 (CN in Fig. 1), the observed trend indicates that UN reporters after 2013 mostly consisted of consumers who used FDA3500B. The contributions of various reporters have changed in the studied decades. Significant proportions of reports in Q1 and Q2 of 2016 were made by PHs (44\%) and UNs (37\%). Given the fact that $72 \%$ of voluntary reports in 2005 were made by healthcare professionals, including PHs and MDs, the contribution of consumers has been steadily on the rise.

\subsection{Contents of Reports: Demographics, Indications, Suspect Drugs, AEs, and Outcomes}

The sex ratio of patients was different between the reporters (Fig. 2a). Reports by consumers tended to have AEs observed in female patients, while reports from healthcare professionals did not show such an imbalance. The average patient age was 55.4, 50.5, 56.9, 52.0, and 52.0 years for reports by CNs, UNs, PHs, MDs, and OTs, respectively (Fig. 2b). Patient outcomes were different according to the occupation of reporters (Fig. 2c). The proportion of disability cases was much higher in reports by $\mathrm{CNs}$ and UNs than those by healthcare professionals. As expected, reports by MDs were likely to include hospitalization much more frequently than those by the other occupations.

Typical profiles of AE reports by each occupation were described in terms of AEs, primary suspect drug, and indication. The types of AEs frequently included were not much different among the reports from different occupations (Table 1). Fatigue, headache, and nausea were reported frequently in the reports from PHs and OTs. Regarding primary suspect drugs, sofosbuvir was most frequently observed in the reports by CNs, PHs, and OTs (Table 2).

Reported indications reflected the indications of primary suspect drugs, although indication was missing in most of the reports (Table 3). In reports with the data available, hepatitis and rheumatoid arthritis were the top two indications reported by CNs, PHs, and OTs. Interestingly, indications in the reports by CNs were different from those by the UNs, although it was supposed that both CNs and UNs indicate consumers, as discussed above. The users of FDA3500 and the users of FDA3500B might therefore be different in background.

\subsection{Report Completeness and Time to Report}

Completeness of reports differed among the occupations [Online Resource 1, see electronic supplementary material (ESM)]. Patient sex and age were reported in most of the reports. For the other items, the reporting rate varied by the type of reporters. Among them, concomitant drug(s) were reported more often in the reports by $\mathrm{CNs}$ and UNs than in those by PHs, MDs, and OTs.

Reports by OTs had the shortest time to report with a median time of 5 days (Fig. 3), while reports by $\mathrm{CNs}$ showed the longest time to report with a median time of 
Fig. 2 Distribution of reports grouped based on patient sex (a), age every 10 years (b), and patient outcome (c). The definition for seriousness of patient outcome in this paper followed the description in the International Conference on Harmonization (ICH) E2 guideline. $C A$ congenital anomaly, $C N$ consumer, $D E$ death, $\mathrm{DS}$ disability, $\mathrm{HO}$ hospitalization-initial or prolonged, $L T$ life-threatening, $M D$ physician, $N S$ non-serious, $O S$ other serious (important medical event), $O T$ other healthcare professional, $P H$ pharmacist, $R I$ required intervention to prevent permanent impairment/damage, $U N$ unknown occupation reporter. ${ }^{*} p<0.05$ (Chi square test for the ratio of reports with a female patient to those with a male patient vs $\mathrm{CN}) ;{ }^{*} p<0.05$ (vs UN)

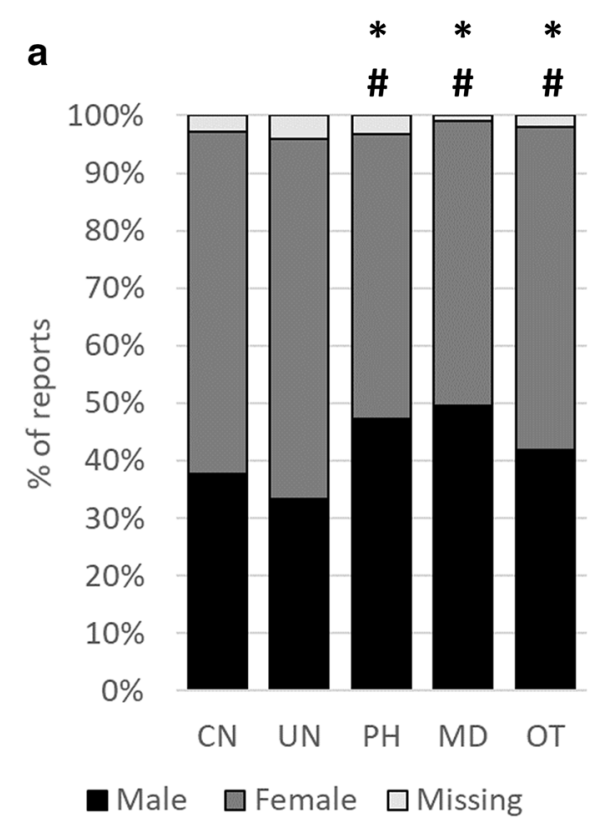

b

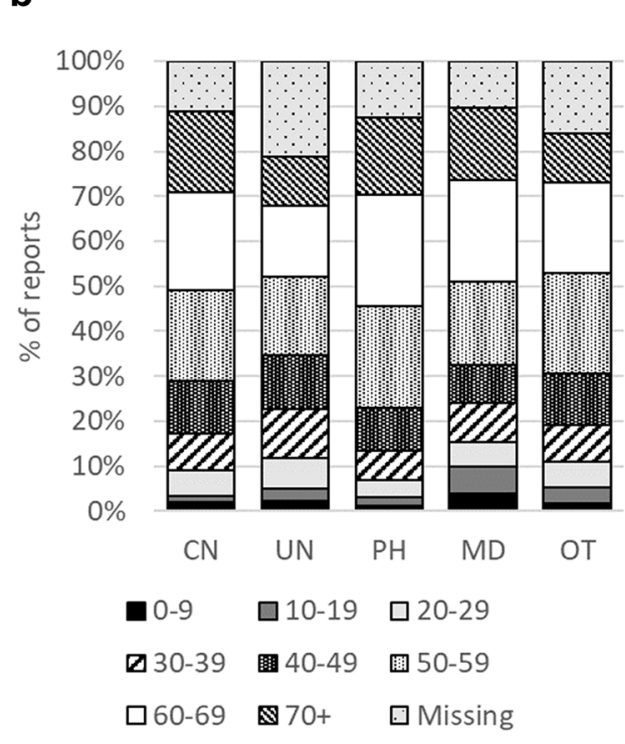

C

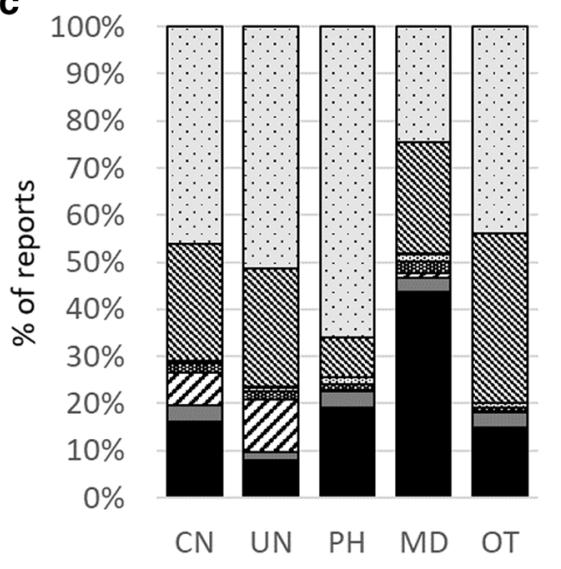

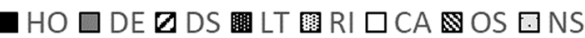

22 days. The time to report by $\mathrm{CNs}$ seemed longer than that by UNs, suggesting that consumers may use the two reporting forms differently, or that the users of each form are somewhat different. The median time for PHs, MDs, and UNs was 9.5, 15, and 15 days, respectively. Time to report was longer in serious cases than in non-serious cases except for the reports by MDs.

We also examined how time to report varied among the occupations depending on whether the AE was known or not, and on whether the primary suspect drug had experienced safety labeling changes (Fig. 4, Online Resource 2 and 3, see ESM). In all the reports by PHs, the time to report for known AEs was shorter by 1 day (median) than that for unknown AEs. In the reports with serious outcome, PHs reported known ADRs slowly, and OTs reported them rather fast.
However, the time to report in consumers' reports was not affected by seriousness of outcomes. CNs submitted AEs where the primary suspect drug had experienced safety labeling changes in the black box warning sections earlier than other AEs (Online Resource 3, see ESM). In contrast, PHs and OTs submitted the AEs earlier when the primary suspect drug had labeling changes in the warning or precaution sections, but not in the black box warning.

\section{Discussion}

Our analysis showed that consumers' reports, which have accounted for a significant portion of recent increases in the number of voluntary reports, seem to have information that 
Table 1 Top 10 MedDRA preferred terms (PTs) for AEs ranked by the most frequently reported by each type of reporter

\begin{tabular}{|c|c|c|c|c|c|c|c|c|c|c|}
\hline & $\mathrm{CN}$ & $\begin{array}{l}\text { No. of } \\
\text { reports }\end{array}$ & UN & $\begin{array}{l}\text { No. of } \\
\text { reports }\end{array}$ & $\mathrm{PH}$ & $\begin{array}{l}\text { No. of } \\
\text { reports }\end{array}$ & MD & $\begin{array}{l}\text { No. of } \\
\text { reports }\end{array}$ & OT & $\begin{array}{l}\text { No. of } \\
\text { reports }\end{array}$ \\
\hline 1 & Headache & 43 & Headache & 404 & Fatigue & 808 & Nausea & 54 & Fatigue & 195 \\
\hline 2 & Nausea & 42 & Fatigue & 376 & Headache & 622 & $\begin{array}{l}\text { Product } \\
\text { substitution } \\
\text { issue }\end{array}$ & 54 & Headache & 159 \\
\hline 3 & Dizziness & 37 & Dizziness & 347 & Nausea & 417 & Pyrexia & 48 & Nausea & 126 \\
\hline 4 & Dyspnea & 37 & Nausea & 345 & Diarrhea & 304 & Drug ineffective & 43 & Diarrhea & 77 \\
\hline 5 & Fatigue & 37 & $\begin{array}{l}\text { Drug } \\
\text { ineffective }\end{array}$ & 316 & Rash & 221 & Diarrhea & 41 & Rash & 63 \\
\hline 6 & Diarrhea & 34 & Pain & 313 & Dizziness & 189 & Fatigue & 38 & $\begin{array}{l}\text { Product } \\
\text { substitution } \\
\text { issue }\end{array}$ & 62 \\
\hline 7 & Pain & 27 & Arthralgia & 312 & Insomnia & 181 & Dizziness & 33 & Dizziness & 54 \\
\hline 8 & Asthenia & 23 & $\begin{array}{l}\text { Blood glucose } \\
\text { increased }\end{array}$ & 296 & Dyspnea & 178 & Seizure & 33 & Insomnia & 47 \\
\hline 9 & Vomiting & 22 & $\begin{array}{l}\text { Pain in } \\
\text { extremity }\end{array}$ & 288 & Vomiting & 178 & Vomiting & 33 & Vomiting & 46 \\
\hline 10 & $\begin{array}{l}\text { Chest } \\
\text { pain }\end{array}$ & 19 & Insomnia & 257 & Pruritus & 138 & Headache & 32 & Drug ineffective & 38 \\
\hline
\end{tabular}

$A E$ adverse event, $C N$ consumer, $M D$ physician, $O T$ other healthcare professional, $P H$ pharmacist, $U N$ unknown occupation reporter

Table 2 Top 10 primary suspect drugs ranked by the most frequently reported by each type of reporter

\begin{tabular}{|c|c|c|c|c|c|c|c|c|c|c|}
\hline & $\mathrm{CN}$ & $\begin{array}{l}\text { No. of } \\
\text { reports }\end{array}$ & UN & $\begin{array}{l}\text { No. of } \\
\text { reports }\end{array}$ & $\mathrm{PH}$ & $\begin{array}{l}\text { No. of } \\
\text { reports }\end{array}$ & MD & $\begin{array}{l}\text { No. of } \\
\text { reports }\end{array}$ & OT & $\begin{array}{l}\text { No. of } \\
\text { reports }\end{array}$ \\
\hline 1 & Sofosbuvir & 71 & Metformin & 704 & Sofosbuvir & 1250 & Cisplatin & 55 & Sofosbuvir & 345 \\
\hline 2 & Ritonavir & 26 & Levonorgestrel & 314 & Everolimus & 366 & Temozolomide & 41 & Ribavirin & 78 \\
\hline 3 & Metformin & 22 & Levofloxacin & 287 & Warfarin & 245 & Cyclophosphamide & 39 & Capecitabine & 64 \\
\hline 4 & Capecitabine & 16 & Ciprofloxacin & 201 & Capecitabine & 188 & Carboplatin & 31 & Everolimus & 52 \\
\hline 5 & Everolimus & 12 & Canagliflozin & 129 & Ribavirin & 180 & Lamotrigine & 28 & Emtricitabine & 45 \\
\hline 6 & Ribavirin & 12 & Etonogestrel & 101 & Ustekinumab & 148 & Canagliflozin & 17 & $\begin{array}{l}\text { Tenofovir } \\
\text { disoproxil } \\
\text { fumarate }\end{array}$ & 45 \\
\hline 7 & Apixaban & 11 & Sofosbuvir & 93 & Rivaroxaban & 125 & Bicalutamide & 15 & Methotrexate & 29 \\
\hline 8 & Deferasirox & 11 & $\begin{array}{l}\text { Sodium } \\
\text { chloride }\end{array}$ & 90 & Dasatinib & 106 & Cytarabine & 15 & Tacrolimus & 28 \\
\hline 9 & Ciprofloxacin & 10 & Rivaroxaban & 80 & Deferasirox & 104 & Dexamethasone & 14 & Ivacaftor & 27 \\
\hline 10 & Hydrochlorothiazide & 10 & Lamotrigine & 76 & Tobramycin & 104 & Sofosbuvir & 14 & Temozolomide & 25 \\
\hline
\end{tabular}

$C N$ consumer, $M D$ physician, $O T$ other healthcare professional, $P H$ pharmacist, $U N$ unknown occupation reporter

is not necessarily provided by healthcare professionals. Differences were observed not only in AEs, suspect drugs, and health outcomes, but also in reporting quality and behaviors such as report completeness and time to report.

In the US, the FDA has facilitated voluntary reports by consumers through several activities such as the release of a new consumer-friendly reporting form FDA3500B and the refurbishment of the interface of its online reporting system through the MedWatch program in 2013 [20, 21]. These efforts apparently have achieved an excellent outcome in enhancing voluntary reporting. Figure 1 indicates that the total number of voluntary reports in the US has increased, especially since 2013 , and that consumers seem to have contributed to that increase. This was probably due to encouragement and increased public recognition that patients, as well as healthcare professionals, could report 
Table 3 Top 10 indications ranked by the most frequently reported by each type of reporter

\begin{tabular}{|c|c|c|c|c|c|c|c|c|c|c|}
\hline & $\mathrm{CN}$ & $\begin{array}{l}\text { No. of } \\
\text { reports }\end{array}$ & $\mathrm{UN}$ & $\begin{array}{l}\text { No. of } \\
\text { reports }\end{array}$ & PH & $\begin{array}{l}\text { No. of } \\
\text { reports }\end{array}$ & MD & $\begin{array}{l}\text { No. of } \\
\text { reports }\end{array}$ & OT & $\begin{array}{l}\text { No. of } \\
\text { reports }\end{array}$ \\
\hline 1 & Hepatitis C & 131 & Missing & 2332 & Missing & 1312 & Missing & 342 & Missing & 479 \\
\hline 2 & Missing & 124 & $\begin{array}{l}\text { Urinary tract } \\
\text { infection }\end{array}$ & 153 & Hepatitis C & 688 & $\begin{array}{l}\text { Type } 2 \text { diabetes } \\
\text { mellitus }\end{array}$ & 21 & Hepatitis C & 246 \\
\hline 3 & $\begin{array}{l}\text { Atrial } \\
\text { fibrillation }\end{array}$ & 14 & Sinusitis & 151 & $\begin{array}{l}\text { Chronic } \\
\text { hepatitis } \mathrm{C}\end{array}$ & 372 & Hepatitis C & 18 & $\begin{array}{l}\text { Chronic hepatitis } \\
\text { C }\end{array}$ & 90 \\
\hline 4 & Hypertension & 10 & Contraception & 132 & $\begin{array}{l}\text { Atrial } \\
\text { fibrillation }\end{array}$ & 368 & Diabetes mellitus & 13 & $\begin{array}{l}\text { Product used for } \\
\text { unknown } \\
\text { indication }\end{array}$ & 68 \\
\hline 5 & Breast cancer & 9 & Hypertension & 103 & $\begin{array}{l}\text { Product used for } \\
\text { unknown } \\
\text { indication }\end{array}$ & 345 & $\begin{array}{l}\text { Chronic hepatitis } \\
\quad \text { C }\end{array}$ & 12 & HIV infection & 55 \\
\hline 6 & HIV infection & 8 & Pneumonia & 87 & $\begin{array}{l}\text { Neoplasm } \\
\text { malignant }\end{array}$ & 175 & Contraception & 9 & Cystic fibrosis & 50 \\
\hline 7 & $\begin{array}{l}\text { Product used for } \\
\text { unknown } \\
\text { indication }\end{array}$ & 8 & Depression & 84 & Cystic fibrosis & 149 & Rhinitis allergic & 9 & $\begin{array}{l}\text { Rheumatoid } \\
\text { arthritis }\end{array}$ & 25 \\
\hline 8 & Anxiety & 7 & Bronchitis & 72 & Hypertension & 146 & Atrial fibrillation & 8 & Hypertension & 22 \\
\hline 9 & Pain & 7 & Pain & 69 & Pain & 145 & $\begin{array}{l}\text { Attention deficit/ } \\
\text { hyperactivity } \\
\text { disorder }\end{array}$ & 8 & $\begin{array}{l}\text { Chronic myeloid } \\
\text { leukemia }\end{array}$ & 20 \\
\hline 10 & $\begin{array}{l}\text { Rheumatoid } \\
\text { arthritis }\end{array}$ & 7 & Hepatitis C & 65 & HIV infection & 122 & Epilepsy & 8 & $\begin{array}{l}\text { Attention deficit/ } \\
\text { hyperactivity } \\
\text { disorder }\end{array}$ & 18 \\
\hline
\end{tabular}

$C N$ consumer, $M D$ physician, $O T$ other healthcare professional, $P H$ pharmacist, $U N$ unknown occupation reporter

Fig. 3 Distribution of time to report stratified by each reporter (a) and the seriousness of patient outcome (b). Boxplot with whiskers with maximum 1.5 interquartile range. Any data not included between whiskers are plotted as an outlier with a dot. If the value for the first quartile is zero, the box is not shown. $C N$ consumer, $M D$ physician, $O T$ other healthcare professional, $P H$ pharmacist, $U N$ unknown occupation reporter. $* p<0.05$ (Wilcoxon rank-sum test)

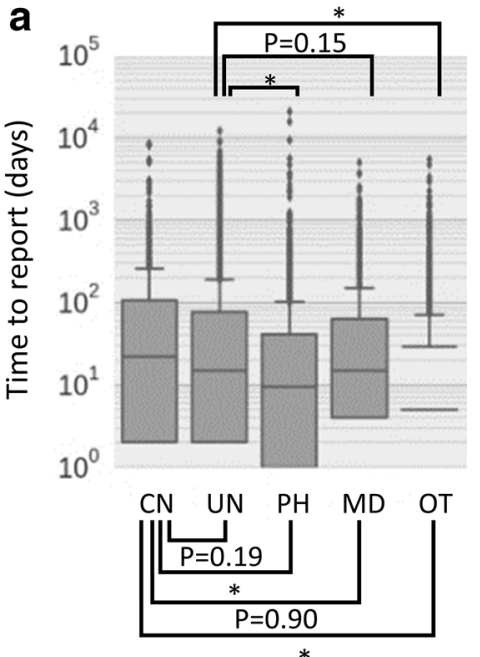

AEs directly to the FDA, especially using the online reporting form.

The increase in the number of reports is beneficial to overall pharmacovigilance activities, but it inevitably draws experts' attention to increasing heterogeneity in the safety database due to diversified reporting sources. Needless to say, statistical inconsistency between different periods is a problem. For data mining purposes, changes in the quality of information must be considered in statistically rigorous ways $[17,35]$. Besides the statistical aspects, safety authorities and drug companies need to decide whether and how we should prioritize concerns for specific safety issues presented by diverse reporters in current public health needs. All these issues make it necessary to 
Fig. 4 Distribution of time to report grouped by whether a report included 'known' drugrelated AEs for all reports (a), reports with serious outcome (b), or reports with non-serious outcome (c). Boxplot with whiskers with maximum 1.5 interquartile range. Any data not included between whiskers are plotted as an outlier with a dot. If the value for the first quartile is zero, the box is not shown. $A E$ adverse event, $C N$ consumer, $M D$ physician, $O T$ other healthcare professional, $\mathrm{PH}$ pharmacist, $U N$ unknown occupation reporter $* p<0.05$ (Wilcoxon rank-sum test)
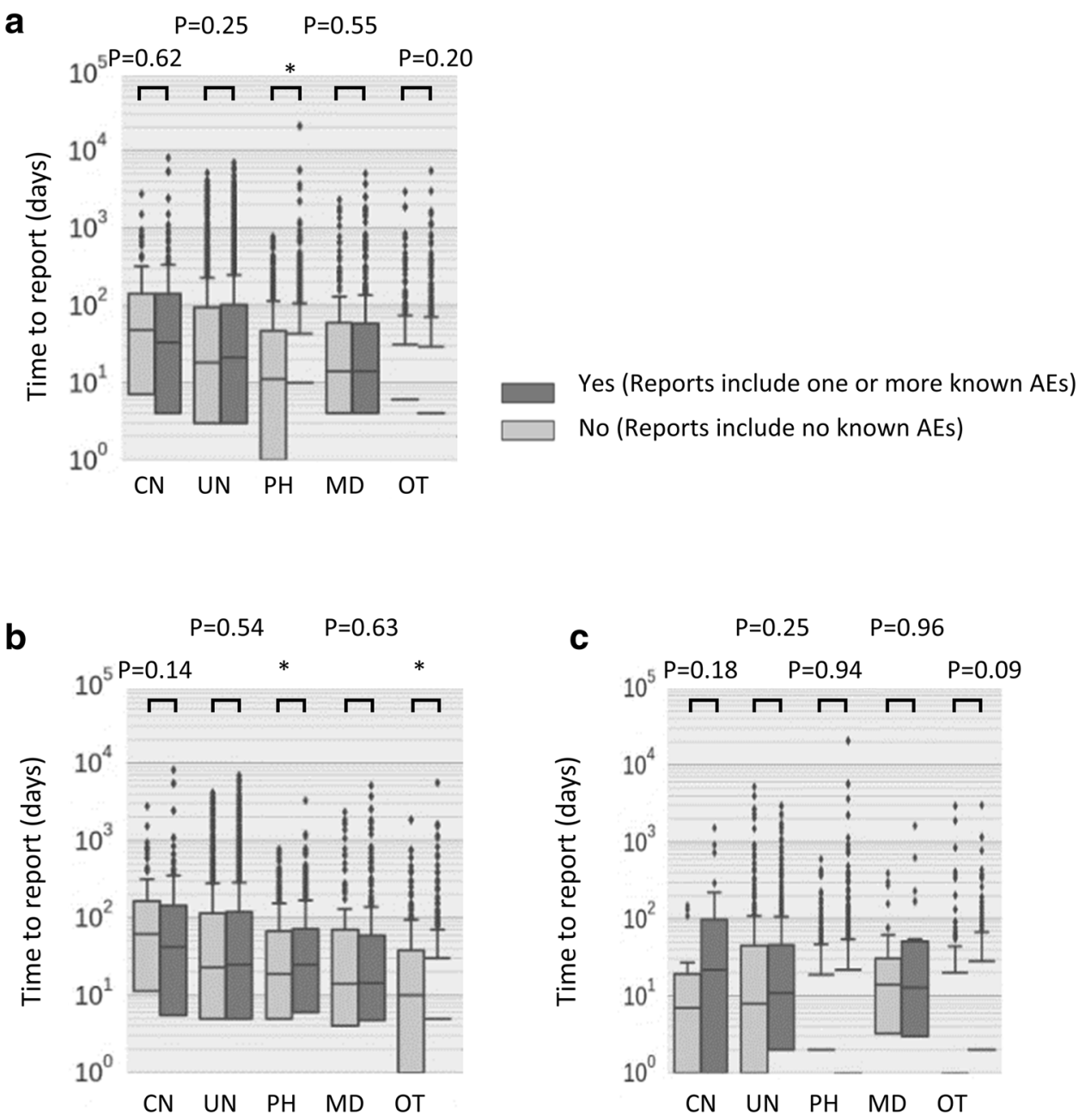

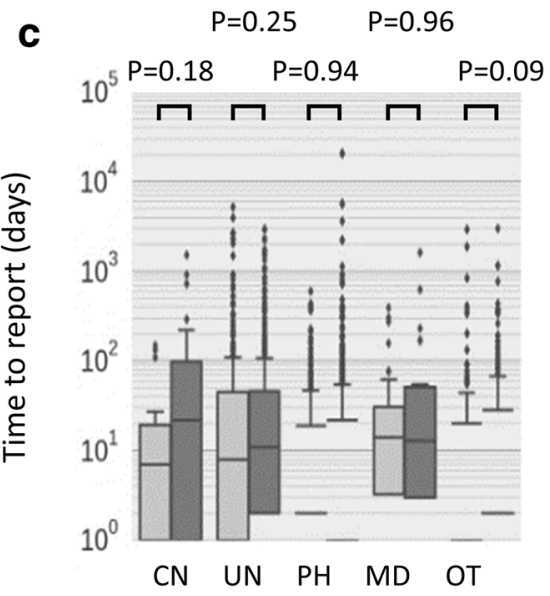

clarify how consumers are different from traditional reporters, mostly healthcare professionals, and also to test whether reports from consumers who choose the new reporting form and routes are different from reports by consumers who choose (or chose) the traditional reporting form.

Our analysis identified several interesting features in US voluntary reports. We found some disparities in patient sex reported by different occupations. Previous studies showed that female patients had a 1.5 - to 1.7 -fold greater risk of developing an ADR, which might be caused by sex differences in pharmacological response [36, 37]. Another recent study presented the same sex disparity and also showed that healthcare professionals tended to report ADRs of male patients more than other types of reporters did [28]. Our results indicate that consumers were more likely to report AEs of female patients than were healthcare professionals (Fig. 2), which was concordant with the findings of previous studies.

Regarding reported patient outcomes, we found that consumers were likely to report AEs that caused disability in patients. In contrast, healthcare professionals rarely reported disability cases. This indicates that reports by consumers can add information about AEs that may not be emphasized by healthcare professionals, and this is consistent with a recent systematic review of the literature on patient reporting [38].

Report completeness has been considered an indicator for well documented AE reports and used for data mining in the World Health Organization's pharmacovigilance database VigiBase [35, 39]. A recent paper showed that voluntary reports have a higher level of report completeness than do reports from drug companies: the completeness of all four items (sex, age, event date, and medical terms) was $86.2 \%$ in serious reports submitted directly to the FDA (i.e., voluntary reports), as compared with $40.4 \%$ in manufacturer-expedited reports and $51.3 \%$ in manufacturer periodic reports, in 2014 [40]. We examined three items (i.e., event date, indication, and route of primary suspect drug) for which data are frequently missing in the FAERS database, and other important items including sex, age, and weight. The report completeness rates for these 
items were almost the same in reports from different reporters, but some findings suggest possible differences in reporters' interest and environment. For example, consumers' reports lack the event date more often than do the other reports (Online Resource 1, see ESM). This may be unsurprising, however, because AEs are detected, recorded, and reported retrospectively based on the records and/or memories available to the reporters. The fact that consumers showed the longest time to report with a median time of 22 days (Fig. 3) may support the finding.

The number of concomitant drugs may reflect reporters' availability of information, and how meticulous the reporters are within real-world constraints. We found that consumers reported more concomitant drugs than did other reporters (Online Resource 1, see ESM). Our preliminary analysis showed that the number of concomitant drugs did not correlate with report completeness for any other items, suggesting that this would not be a quality measure for general purposes.

In general, consumers are thought to have less access to information on drug-related AEs in drug labels than do healthcare professionals. Consumers do not have expertise in pharmacovigilance activities, either. We had expected that previous knowledge about AEs would affect reporting behaviors differently for consumers and healthcare professionals, but our analysis using all the reports did not show a clear difference between the two groups (Fig. 4a). This is basically in line with a conclusion in a previous report that patients can make causality assessment based on the available information [31, 32]. We further examined serious ADR cases and found that known ADRs were reported more slowly than unknown ADRs by PHs, and vice versa by OTs (Fig. 4b). This suggests differences seem to exist among healthcare professionals.

Histories of labeling revisions were associated with observed differences between consumers and healthcare professionals in what and how they are likely to report (Online Resource 2 and 3, see ESM). A possible explanation is that healthcare professionals, and MDs in particular, are less likely to be influenced by prior information about risk and/or more likely to adhere to their own judgement than are consumers. However, it is difficult to discuss the role of prior information and environment based solely on our findings, because there are many confounding factors. The issuance of regulatory alerts, for example, is a possible confounder that has been investigated intensively in many studies [41, 42].

Time to report is an interesting measure of promptness in reporting and may help to describe reporting behaviors. A recent study in the EU showed that median time to report an ADR was approximately 30 days in spontaneous reports, regardless of whether it was reported by patients or healthcare professionals [28]. As shown in Fig. 3a, the median time to report AEs by any reporter in the US was shorter than that in the EU. These differences in time to report are likely to reflect differences in reporting pathways: the voluntary $\mathrm{AE}$ reports in this research were directly submitted to the FDA, while some reports in the EU arrived at the authority via companies or other regulatory agencies. Different reporting times may also be influenced by different cultures and histories surrounding the reporting systems and use of drugs, such as publicity around medicinal products, as a previous report suggested [43]. With respect to suspect drugs, Table 2 indicates that MDs report AEs of oncologic injection drugs (e.g., cisplatin and carboplatin) but consumers do not, which suggests that MDs and consumers report different AEs in different settings, and may explain our observation (at least partly) that time to report was much shorter in MDs than in consumers. It is worthwhile investigating whether this reflects differences in the publicity and maturation of the $\mathrm{AE}$ reporting system between the two regions.

It was also shown that time to report by consumers was longer than that by pharmacists and other healthcare professionals, and almost equal to that by physicians (Fig. 3a). Comparisons between serious and non-serious cases showed that time to report was longer in serious cases than in non-serious cases except in the reports by physicians (Fig. 3b). With regard to whether the $\mathrm{AE}(\mathrm{s})$ were already written in the labels, interesting differences were observed between healthcare professionals in reports with serious outcome(s) (Fig. 4b). Safety labeling changes of primary suspect drugs were associated with the time to report of healthcare professionals but not with those of consumers (Online Resource 3, see ESM). Time to report was associated with which section of labeling (i.e., black box warning, warning, or precaution) had been revised, but in somewhat complicated ways. Interestingly, pharmacists and other healthcare professionals tended to report earlier the primary suspect drug for which safety labeling changes occurred in the warning or precaution sections, but not in the black box warning.

Causalities behind these findings on time to report are complex and beyond our scope, but they probably reflect diversities in the environment where reporters come to experience and/or be aware of AEs and decide to report AEs to the authority. Some consumers may have difficulties in reporting their own AE immediately after their recovery, and need to take time to learn how to report AEs even when they intend to do so. Physicians, who commonly struggle with time conflicts on a daily basis, may face tradeoffs between voluntary and mandatory reporting. Causal determination for AEs did not seem to play a critical role as shown in Fig. 4 and Online Resource 3 (see ESM). This is in line with a previous report that some consumers may be able to identify suspected ADRs 
adequately [31, 32]. To improve efficiency and responsiveness in the current reporting system, we need to consider real-world mechanisms of reporting behaviors. For example, we need to examine the possible influence of communications such as 'Dear Healthcare Provider' letters, which are expected to have substantial impacts on reporting behaviors [41, 42].

Our analysis provided several clues to the heterogeneity in reports from consumers, which were conveyed in one of the two different forms: the traditional form FDA3500 (tagged as ' $\mathrm{CN}$ ' in this paper) and the new form FDA3500B (tagged as 'UN'). It is apparently the latter form that has contributed to the recent increase in the number of $\mathrm{AE}$ reports. Although the traditional form users and the new form users were similar in some aspects (e.g., concomitant drugs, report completeness), they were apparently different in other important aspects including reported outcomes and indications, and time to report. It is quite natural that consumers use forms differently when a new reporting form and/or route is added to traditional forms/routes, and this might be exactly what regulators intended to facilitate. Our results suggest that database users have to be careful about such heterogeneities when combining or pooling reports made using the different forms, even though they were all submitted by 'consumers.'

Finally, we can discuss our findings with reference to previous studies in the EU. As discussed above, the average time to report in the US was much shorter than that in the EU, which may reflect the extent of differences in regulations and reporting pathway(s) [28]. Reporting behaviors in consumers, including promptness to report, may be affected by marketing environments such as directto-consumer advertising [43]. Irrespective of these differences between the US and European countries, we found that consumers' reports in both regions are similar in some important aspects. For example, consumers in both regions tend to report subjective symptoms such as headache, fatigue, and nausea [28]. Avery et al. investigated patient reporting in UK's Yellow Card Scheme and discussed how patient reporting may provide a positive complementary contribution to that of healthcare providers; however, the combination of reports from patients and healthcare providers, when used for the purposes of signal detection through disproportionality analysis, may result in the loss of some information [31, 32]. Our findings in the US lead to similar conclusions to these previous studies in European countries.

This study has several limitations. Because it relied on spontaneous reporting data, all the limitations inherent to spontaneous reporting are applicable. We focused on consumers' reporting, but lack of publicly available information about the choice of reporting forms and reporters' occupations made it difficult to extract data from 'real' consumers. There are no official rules and/or principles on how to handle consumers' reports in publication and data mining, and our analysis had to be done under such uncertainties. Our analysis did not cover mandatory reports, which limits the generalizability of our results. A significant portion of $\mathrm{AE}$ reporting is done as mandatory reporting, and drug companies play a key role in it. Healthcare professionals choose reporting route(s) considering types and seriousness of AEs, applicable rules, and many other factors including opportunity costs of reporting. These considerations affect how healthcare professionals report AEs voluntarily. Further studies are needed to shed light on such broader aspects and examine the system as a whole.

\section{Conclusion}

Our analysis of voluntary AE reports in the US FAERS database has shown the characteristics of spontaneous reporting in the US. Voluntary reports tended to include AEs related to subjective symptoms, as in some previous studies on patient reporting in the EU. Voluntary reports by consumers seemed to be different from reports by healthcare professionals in demographics and outcomes of patients, and suspect drugs. They were also different in report completeness and time to report, which may reflect concerns and environments that are specific to each type of reporter. Consumers' choice of voluntary reporting forms may be worth studying further. These findings suggest that the heterogeneities should be addressed appropriately when using spontaneous reports, especially in the context of international comparison.

Acknowledgements We would like to thank Japan Pharmaceutical Information Center (JAPIC) for permitting our use of the JAPIC AERS database under a collaborative research contract. We would like to thank Editage (http://www.editage.jp) for English language editing.

\section{Compliance with Ethical Standards}

Funding This study was funded by a Japanese government-based Grant-in-aid from the Ministry of Education, Culture, Sports, Science, and Technology, Tokyo, Japan (Grant KAKENHI: 26460215). The funders had no role in study design, data collection and analysis, decision to publish, or preparation of the manuscript.

Conflict of interest Tadashi Toki was employed by Daiichi Sankyo Co. Ltd during the study period. Shunsuke Ono declares no conflict of interest.

Ethics approval This study was performed on anonymized patient data and ethics committee approval was therefore not required. 
Open Access This article is distributed under the terms of the Creative Commons Attribution-NonCommercial 4.0 International License (http://creativecommons.org/licenses/by-nc/4.0/), which permits any noncommercial use, distribution, and reproduction in any medium, provided you give appropriate credit to the original author(s) and the source, provide a link to the Creative Commons license, and indicate if changes were made.

\section{References}

1. US Food and Drug Administration. Breakthrough therapy designation. 2017. http://www.fda.gov/forpatients/approvals/fast/ ucm405397.htm. Accessed 14 Nov 2017.

2. European Medicines Agency. PRIME: priority medicines. 2017. http://www.ema.europa.eu/ema/index.jsp?curl=pages/regulation/ general/general_content_000660.jsp\&mid=WC0b01ac05809f84 39. Accessed 14 Nov 2017.

3. Minister of Health, Labour and Welfare. Strategy of SAKIGAKE. 2017. http://www.mhlw.go.jp/english/policy/health-medical/ pharmaceuticals/140729-01.html. Accessed 14 Nov 2017.

4. Kwok M, Foster T, Steinberg M. Expedited programs for serious conditions: an update on breakthrough therapy designation. Clin Ther. 2015;37(9):2104-20.

5. Mostaghim SR, Gagne JJ, Kesselheim AS. Safety related label changes for new drugs after approval in the US through expedited regulatory pathways: retrospective cohort study. BMJ. 2017;358:j3837.

6. US Food and Drug Administration. Questions and answers on FDA's adverse event reporting system (FAERS). 2017. https:// www.fda.gov/Drugs/GuidanceComplianceRegulatoryInforma tion/Surveillance/AdverseDrugEffects/default.htm. Accessed 20 June 2017.

7. US Food and Drug Administration. Medical product safety information. 2017. https://www.fda.gov/Safety/MedWatch/ SafetyInformation/default.htm. Accessed 26 Aug 2017.

8. Matsui T, Umetsu R, Kato Y, et al. Age-related trends in injection site reaction incidence induced by the tumor necrosis factor-alpha (TNF-alpha) inhibitors etanercept and adalimumab: the food and drug administration adverse event reporting system, 2004-2015. Int J Med Sci. 2017;14(2):102-9.

9. Fujimoto M, Kanou M, Hosomi K, Takada M. Angiotensin receptor blockers and the risk of cancer: data mining of a spontaneous reporting database and a claims database. Int J Clin Pharmacol Ther. 2017;55(4):295-303.

10. Blau JE, Tella SH, Taylor SI, Rother KI. Ketoacidosis and SGLT2 inhibitor treatment: analysis of FAERS data. Diabetes Metab Res Rev. 2017;33(8):e2924.

11. Kimura G, Kadoyama K, Brown JB, et al. Antipsychotics-associated serious adverse events in children: an analysis of the FAERS database. Int J Med Sci. 2015;12(2):135-40.

12. Xu R, Wang Q. Automatic signal extraction, prioritizing and filtering approaches in detecting post-marketing cardiovascular events associated with targeted cancer drugs from the FDA adverse event reporting system (FAERS). J Biomed Inform. 2014;47:171-7.

13. Iyer G, Marimuthu SP, Segal JB, Singh S. An algorithm to identify generic drugs in the FDA adverse event reporting system. Drug Saf. 2017;40(9):799-808.

14. Rahman MM, Alatawi Y, Cheng N, et al. Comparison of brand versus generic antiepileptic drug adverse event reporting rates in the US food and drug administration adverse event reporting system (FAERS). Epilepsy Res. 2017;135:71-8.
15. Nagashima T, Shirakawa H, Nakagawa T, Kaneko S. Prevention of antipsychotic-induced hyperglycaemia by vitamin D: a data mining prediction followed by experimental exploration of the molecular mechanism. Sci Rep. 2016;6:26375.

16. Zhao S, Nishimura T, Chen Y, et al. Systems pharmacology of adverse event mitigation by drug combinations. Sci Transl Med. 2013;5(206):206ra140.

17. Lindquist M. Data quality management in pharmacovigilance. Drug Saf. 2004;27(12):857-70.

18. Stergiopoulos S, Brown CA, Felix T, Grampp G, Getz KA. A survey of adverse event reporting practices among US healthcare professionals. Drug Saf. 2016;39(11):1117-27.

19. Alatawi Yasser M, Hansen Richard A. Empirical estimation of under-reporting in the US food and drug administration adverse Event Reporting system (FAERS). Expert Opin Drug Saf. 2017;16(7):761-7.

20. Rose BJ, Fritsch BF. FDA's MedWatch program turns 20: what's new? PharmacyToday Web site. 2013. http://pharmacytoday.org/ article/S1042-0991(15)31139-7/pdf. Accessed 12 June 2017.

21. Hamburg MA. MedWatch: improving on 20 years of excellence. FDA Voice Web site. 2013. https://blogs.fda.gov/fdavoice/index. php/2013/06/medwatch-improving-on-20-years-of-excellence/. Accessed 12 Jun 2017.

22. Rowin EJ, Lucier D, Pauker SG, Kumar S, Chen J, Salem DN. Does error and adverse event reporting by physicians and nurses differ? Jt Comm J Qual Patient Saf. 2008;34(9):537-45.

23. Pushkin R, Frassetto L, Tsourounis C, Segal ES, Kim S. Improving the reporting of adverse drug reactions in the hospital setting. Postgrad Med. 2010;122(6):154-64.

24. Glenn DG. Big data and pharmacovigilance: the role of oncology nurses. Clin J Oncol Nurs. 2016;20(5):478-80.

25. Andrew Herxheimer, Rose Crombag and Teresa Leonardo Alves. Direct patient reporting of adverse drug reactions. 2010. https:// consumers.cochrane.org/sites/consumers.cochrane.org/files/public/ uploads/10\%20May\%202010\%20Report\%20Direct\%20Patient\% 20Reporting\%20of\%20ADRs.pdf. Accessed 20 Mar 2018.

26. Weigmann K. Consumer reporting of adverse drug reactions: systems that allow patients to report side effects of the drugs they are taking have yielded valuable information for improving drugs safety and health care. EMBO Rep. 2016;17(7):949-52.

27. Scurti V, Romero M, Tognoni G. A plea for a more epidemiological and patient-oriented pharmacovigilance. Eur J Clin Pharmacol. 2012;68(1):11-9.

28. Banovac M, Candore G, Slattery J, et al. Patient reporting in the EU: analysis of EudraVigilance data. Drug Saf. 2017;40(7):629-45.

29. Rolfes L, van Hunsel F, van der Linden L, Taxis K, van Puijenbroek E. The quality of clinical information in adverse drug reaction reports by patients and healthcare professionals: a retrospective comparative analysis. Drug Saf. 2017;40(7):607-14.

30. Leone R, Moretti U, D'Incau P, et al. Effect of pharmacist involvement on patient reporting of adverse drug reactions: first Italian study. Drug Saf. 2013;36(4):267-76.

31. Avery AJ, Anderson C, Bond CM, et al. Evaluation of patient reporting of adverse drug reactions to the UK 'yellow card scheme': literature review, descriptive and qualitative analyses, and questionnaire surveys. Health Technol Assess. 2011;15(20):1-234.

32. Krska J, Anderson C, Murphy E, Avery AJ. How patient reporters identify adverse drug reactions: a qualitative study of reporting via the UK yellow card scheme. Drug Saf. 2011;34(5):429-36.

33. Downloading data (SIDER4.1). Sider side effect resources web site. 2015. http://sideeffects.embl.de/download/. Accessed 18 Apr 2017.

34. US Food and Drug Administration. Drug safety labeling changes. 2018. https://www.fda.gov/Safety/MedWatch/SafetyInformation/ 
Safety-RelatedDrugLabelingChanges/default.htm. Accessed 21 Mar 2018.

35. Caster O, Juhlin K, Watson S, Noren GN. Improved statistical signal detection in pharmacovigilance by combining multiple strength-of-evidence aspects in vigiRank. Drug Saf. 2014;37(8):617-28.

36. Rademaker M. Do women have more adverse drug reactions? Am J Clin Dermatol. 2001;2(6):349-51.

37. Anderson GD. Gender differences in pharmacological response. Int Rev Neurobiol. 2008;83:1-10.

38. Inacio $\mathrm{P}$, Cavaco A, Airaksinen $\mathrm{M}$. The value of patient reporting to the pharmacovigilance system: a systematic review. Br J Clin Pharmacol. 2017;83(2):227-46.

39. Bergvall T, Noren GN, Lindquist M. vigiGrade: a tool to identify well-documented individual case reports and highlight systematic data quality issues. Drug Saf. 2014;37(1):65-77.
40. Moore TJ, Furberg CD, Mattison DR, Cohen MR. Completeness of serious adverse drug event reports received by the US food and drug administration in 2014. Pharmacoepidemiol Drug Saf. 2016;25(6):713-8.

41. Pariente A, Gregoire F, Fourrier-Reglat A, Haramburu F, Moore $\mathrm{N}$. Impact of safety alerts on measures of disproportionality in spontaneous reporting database. Drug Saf. 2007;30(10):891-8.

42. Hoffman KB, Demakas AR, Dimbil M, Tatonetti NP, Erdman CB. Stimulated reporting: the impact of US food and drug administration-issued alerts on the adverse event reporting system (FAERS). Drug Saf. 2014;37(11):971-80.

43. Ventola CL. Direct-to-consumer pharmaceutical advertising: therapeutic or toxic? Pharm Ther. 2011;36(10):669-74. 